\title{
Harmonic Suppression Strategy in Single-phase SPWM Based on Mixed Integer Nonlinear Programming
}

\author{
Lubin $\mathrm{Wu}^{1}$, Ziquan Liang ${ }^{1}$, Weihua $\mathrm{Li}^{1} \&$ Xiaoteng Wang ${ }^{2}$ \\ ${ }^{1}$ College of Electrical Engineering, Jinan University, Zhuhai, China \\ ${ }^{2}$ College of Intelligent Science and Engineering, Jinan University, Zhuhai, China \\ Correspondence: Weihua Li, College of Electrical Engineering, Jinan University, No. 206, Qianshan Road, \\ Xiangzhou District, Zhuhai City, Guangdong Province, China. Tel: 86-134-1137-3512. E-mail: \\ liweihua115@163.com
}

Received: March 1, 2019

Accepted: April 1, 2019

Online Published: April 14, 2019

doi:10.5539/mas.v13n5p22

URL: https://doi.org/10.5539/mas.v13n5p22

\begin{abstract}
In single-phase bipolar SPWM inverter circuit, the output voltage contains high content of harmonic components. To solve this problem, we build a model of output voltage in single-phase SPWM bipolar inverter based on SPWM theory and Fourier series theory. Through theoretical analysis, calculation and plotting, we work out the distribution of harmonic in output voltage, investigate the relationship between amplitude modulation (AM) depth and harmonic components and finally determine a proper amplitude modulation depth to efficiently reduce harmonic components. To further reduce harmonic components of output voltage, we use LC low-pass filter. Then, based on the mathematical model of the filter and the distribution law of harmonics, we establish a mixed integer nonlinear programming to fully suppress harmonics and reduce switching losses. After searching for two times based on genetic algorithm, we work out the best frequency-modulation ratio and LC low-pass filter parameter. Finally in simulation, total harmonic distortion is reduced from 213.47 percent to 0.16 percent after harmonic suppression strategy implemented. The result has proved the effectiveness of the harmonic suppression strategy we applied, which can be used as a reference for output voltage harmonic elimination in single-phase SPWM inverter.
\end{abstract}

Keywords: harmonic suppression, LC low-pass filter, mixed integer nonlinear programming, genetic algorithm

\section{Introduction}

As power electronics technology rapidly develops, SPWM inverter becomes widely used in industry, transportation, energy and aeronautic and astronautics. To satisfy various demands in practical applications, people would like output voltage, current, power and frequency of inverters can be effectively and flexibly controlled. For instance, some motor systems have strict requirements for the distortion rate of output voltage's sinusoidal waveform.

Featuring low output harmonics and high precision, sinusoidal pulse-width modulation (SPWM) is the most important as well as the most widely used modulation method in inverter power (Blanco, 2017). SPWM wave is a kind of wave whose pulse-width changes sinusoidally and it is equivalent to a sine wave. Sinusoidal pulse-width modulation (SPWM) controls switching signals in inverter circuits according to SPWM wave and makes the area of actual output impulse voltage equal to that of expect output sine wave in corresponding interval. For SPWM, the frequencies and amplitudes of output voltage in inverter circuits are changed by modulating the frequencies and amplitudes of the output waveform (Hannan, Aslam \& Ghayur, 2018).

Because properties of inverters depend to a large extent on harmonics in output voltage (Lin, 2006), detailed and exact analysis on output harmonics in SPWM inverter as well as effective suppression method is necessary. And a number of scholars have published a lot of researches on harmonic suppression. $\mathrm{Wu}, \mathrm{Y}$ et al propose an improved repetitive controller based on Lagrangian interpolation method to suppress harmonics (Wu, Song, Li \& Chen, 2018). But they don't study the relationship between amplitude modulation depth and the content of harmonics. The circuit topology of single-stage isolated charging/discharging DC-AC converter designed by Hanchao, Z and Daolian, C do well in suppressing second-harmonics (Hanchao \& Daolian, 2017). XU, S. G et al eliminate harmonics with random modulation to distribute output harmonic energy of inverter power more evenly (S. XU, 
J. XU \& CAO, 2010). ZHOU, K. L et al quantize pulse width difference sectionally to improve calculating method of SPWM signals. However, they ignore to analyze distribution of harmonic amplitude of each frequency (ZHOU, LIN \& HAN, 2003). Besides, multi-level harmonic elimination modulation method (Rahman et al., 2018) and specific harmonic elimination pulse modulation method are also kinds of harmonic suppression methods.

In order to improve the properties of SPWM inverter, based on a review of relevant domestic and foreign researches, this article put forwards a harmonic suppression strategy in single-phase SPWM based on mixed integer nonlinear programming from the perspective of industrial application. Also, this article thoroughly simulates and analyzes this strategy's effect on eliminating harmonics in output voltage.

\section{Method}

\subsection{Harmonic Analysis in Single-phase Bipolar SPWM Inverter}

Single-phase bipolar SPWM inverter circuit usually consists of four shunt circuits of IGBT and diodes, which is showed in the left picture in Figure 1. By comparison controlling modulation signal and carrier signal, we can control IGBT's on-off state and get expect output wave. Unipolar SPWM and bipolar SPWM use different comparison controlling methods to control the two signals. Bipolar SPWM takes expect output wave as modulation signal $u_{r}$ and triangular wave signal with positive and negative polarities as carrier signal $u_{c}$. If $u_{r}>u_{c}$, the output of SPWM inverter is postive polarity voltage $U_{d}$ and otherwise negative polar voltage $-U_{d}$. However, according to the output wave of voltage $u_{o}$ in the right picture in Figure 1, output voltage is composed by a lot of square wave and contains much high-order harmonic components. As a result, harmonic analysis on SPWM wave is necessary (Zhaoan \& Jun, 2000).

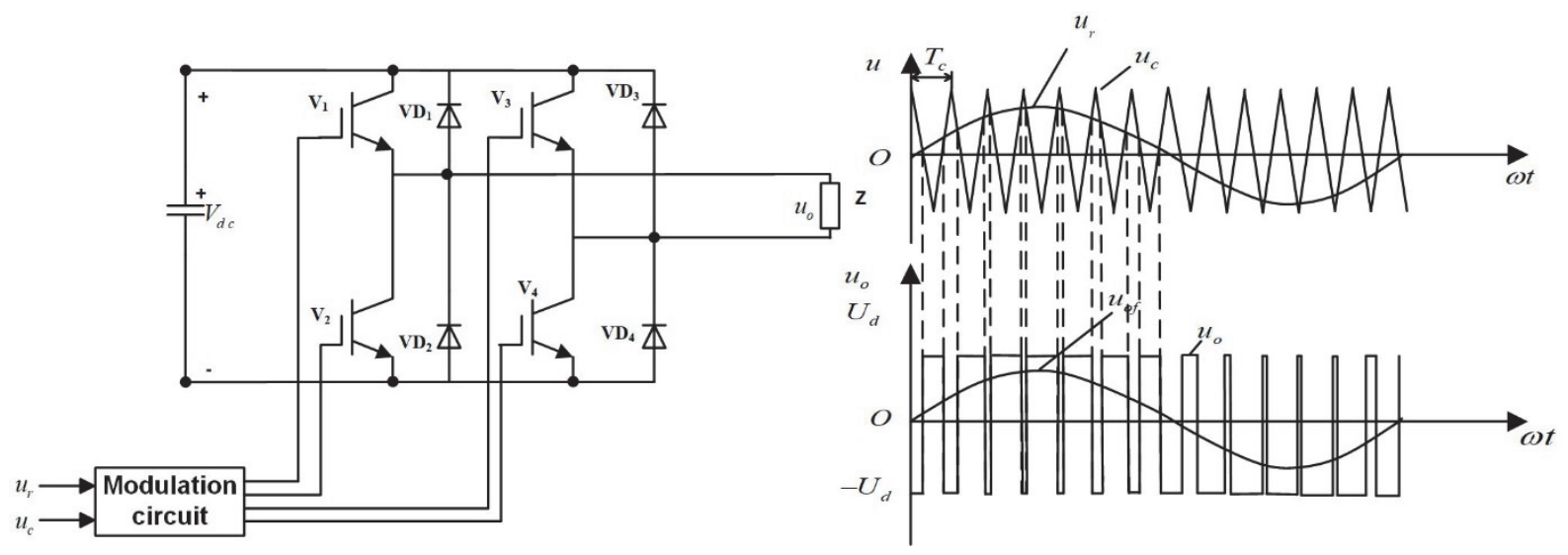

Figure 1. Single-phase SPWM inverter circuit (left) and output voltage waveform (right)

To make harmonics easy to analyze, we set modulation signal which is also the sinusoidal waveform signal as

$$
u_{r}=U_{r m} \sin \left(w_{r} t+\varphi\right)
$$

Where $U_{r m}$ is the peak of modulation signal, $w_{r}$ is the angular frequency and $\varphi$ is the phase of modulation signal.

Set the peak of carrier signal as $U_{c m}\left(U_{c m} \geq U_{r m}\right)$, angular frequency of carrier signal as $w_{c}$ and its period as $T_{c}$. Then the frequency modulation index is

$$
P=\frac{w_{c}}{w_{r}}
$$

And the amplitude modulation depth is

$$
M=\frac{U_{r m}}{U_{c m}}
$$

Dividing carrier signal in a modulation signal period into k segments, each of which intersects with $\mathrm{x}$-coordinate, we obtain the expression of carrier signal. 


$$
u_{c}= \begin{cases}-\frac{4 U_{c m}}{T_{c}}\left(t-\frac{T_{c}}{4}-\frac{(k-1)}{2} T_{c}\right) & k \text { is odd number } \\ \frac{4 U_{c m}}{T_{c}}\left(t-\frac{3 T_{c}}{4}-\frac{(k-2)}{2} T_{c}\right) & k \text { is even }\end{cases}
$$

Combine formula 1 and 4 , the time $\left(t_{m}\right)$ of controlling IGBT with SPWM can be figured out. According to Fourier series theory, the harmonic angular frequency $w$, fundamental amplitude $U_{b}$ and harmonic voltage amplitude $U$ of each harmonic component of the output voltage $u_{o}$ can be obtained (Fengjun, 2002).

$$
\begin{gathered}
w=n w_{c} \pm m w_{r} \\
U_{b}=M U_{d} \\
U=\frac{4 U_{d}}{n \pi} J_{m}\left(\frac{M n \pi}{2}\right)
\end{gathered}
$$

Where $J_{m}$ is Bessel function to the $m^{\text {th }}$ power. $m$ is equal to $0,2,4, \ldots, \mathrm{K}$ when $n$ is $1,3,5, \ldots, \mathrm{K}$, and equals $1,3,5, \ldots, \mathrm{K}$ when $n$ is $2,4,6, \ldots, \mathrm{K}$.

In formula 6, fundamental amplitude $U_{b}$ is direct proportional to amplitude modulation depth $M$. Circuit with a larger amplitude modulation depth $M$ has a larger fundamental amplitude $U_{b}$ in output voltage. And according to formula 7, harmonic amplitude $U$ in output voltage is related to amplitude modulation depth $M$. Given a fixed $M$ and variable $n, m$, how output voltage's harmonic amplitude changes with harmonic angular frequency is discussed. With $U_{d}=1, M=0.8$, integer $m$ ranging within $[0,599]$ and integer $n$ ranging within $[1,40]$, harmonic frequency is $n w_{c} \pm m w_{r}$ and the distribution of harmonic amplitude $\mathrm{U}$ is showed in Figure 2.

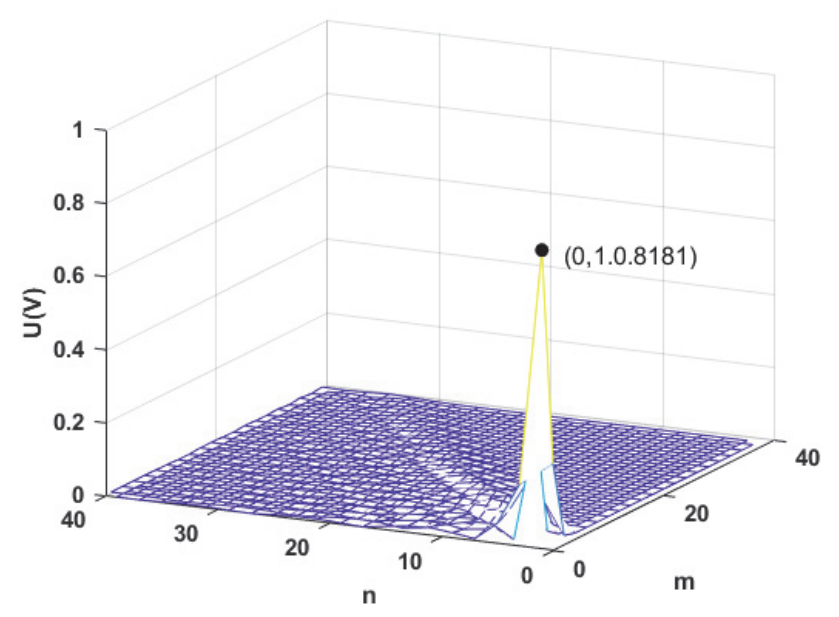

Figure 2. The relationship between harmonic amplitude and harmonic frequency

Figure 2 shows that output voltage's largest harmonic amplitude correspond to a harmonic frequency of $1 * w_{c} \pm 0 * \mathrm{w}_{r}$. Given the value of $M \quad 0.1,0.2,0.3,0.4,0.5,0.6,0.7,0.8,0.9$ separately, the harmonic component whose frequency is $w_{c}$ has the largest amplitude. It can be referred that the harmonic component whose frequency is $w_{c}$ accounts for the largest part among all harmonic components and has the most impact on output voltage's property.

\subsection{Comprehensive Method for Single-phase Bipolar SPWM Harmonic Suppression}

\subsubsection{The Determination of Amplitude Modulation Depth $M$}

For different amplitude modulation depths $M$, the sum of the amplitude of each harmonic component is different.

$$
U_{\text {sum }}=\sum_{m=0}^{\infty} \sum_{n=1}^{\infty} \frac{4 U_{d}}{n \pi} J_{m}\left(\frac{M n \pi}{2}\right)
$$


In formula 8 , if $n$ equals $1,3,5, \ldots \mathrm{K}, m$ is $0,2,4, \ldots \mathrm{K}$, and if $n$ equals $2,4,6, \ldots \mathrm{K}$ then $m$ is $1,3,5, \ldots$ K.

In the research in this article, we choose IGBT which has a switching frequency no higher than $30 \mathrm{kHz}$ so that frequency modulation index $\mathrm{P}$ is not larger than 600 . Also, harmonic component with an angular frequency of $w_{c}$ is the largest component which most drastically affects output voltage and is easy to filtered away as high-order harmonics. As a result, we ignore high-order harmonics in the range of $m \geq 600, n>40$ and select $m$ from integers in $[0,599]$ and $n$ from integers in $[1,40]$. And we get the sum of harmonic amplitude similar to $\sum_{m=0}^{599} \sum_{n=1}^{40} \frac{4 U_{d}}{n \pi} J_{m}\left(\frac{M n \pi}{2}\right)$. Given $U_{d}=50 \mathrm{~V}$ and amplitude modulation depth $M$ ranging in [0,1], relationships between $\mathrm{M}$ and the sum $U_{\text {sum }}$, fundamental amplitude $U_{b}$ and harmonic amplitude $U_{w_{c}}$ corresponding to the angular frequency $w_{c}$ are showed in Figure 3.

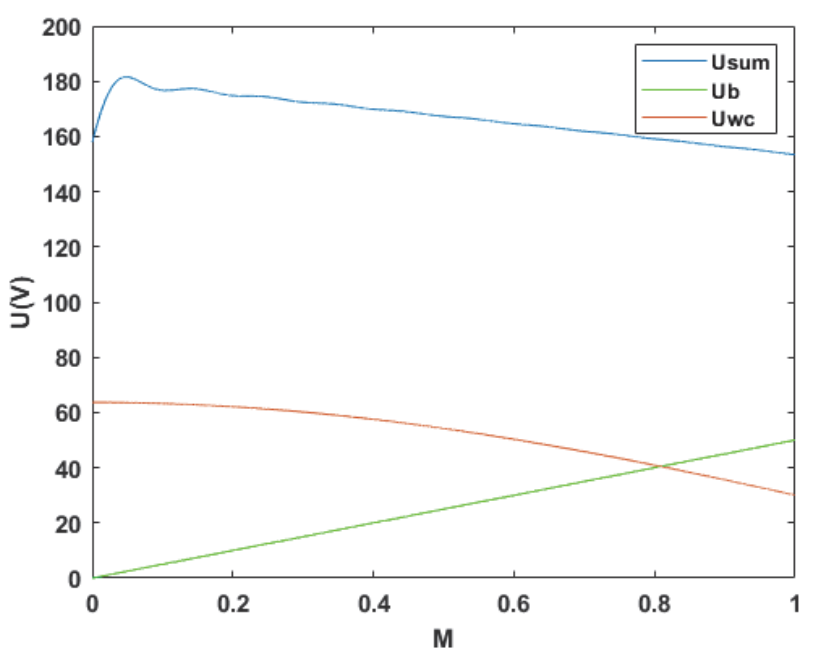

Figure 3. Influence of amplitude modulation depth (M) on the $U_{\text {sum }}, U_{b}$ and $U_{w_{c}}$

From Figure 3, we know that fundamental amplitude $U_{b}$ grows linearly as amplitude modulation depth $M$ goes up and that the sum of harmonic amplitude $U_{\text {sum }}$ goes up if $M$ is less than 0.05 but the opposite if $M$ is larger than 0.05. And $U_{\text {sum }}$ is always larger than fundamental amplitude. As for harmonic component with an angular frequency of $w_{c}$, its amplitude $U_{w_{c}}$ declines as $M$ rises. $\mathrm{M}=0.81$ is the demarcation point where $U_{w_{c}}$ is larger than fundamental amplitude before but less then fundamental amplitude after. Therefore, when $\mathrm{M}$ equals 1 , the sum of harmonic amplitude $U_{\text {sum }}$ and $U_{w_{c}}$, the amplitude of the harmonic component with an angular frequency of $w_{c}$, reach their minimum values. And this is the best condition for harmonic suppression.

\subsubsection{Establishment of Mixed Integer Nonlinear Programming Model}

Properly increasing frequency modulation index $P$ helps raising the value of carrier signal frequency $w_{c}$ and makes harmonic components distributed in higher frequency band, which is good for the filtering of high order harmonics. Therefore, after selecting a proper amplitude modulation depth $M=1$, we analyze the distribution of SPWM harmonics and select the most influential harmonics as the focus of harmonic suppression. Then we establish a mixed integer nonlinear programming model to determine the frequency modulation index $P$ and the parameter of low-pass filter, thus effectively eliminate harmonics.

\subsubsection{Constraint of Low-Pass Filter's cut-off Frequency}

When $M=1, U_{d}=50 \mathrm{~V}$, fundamental frequency $f_{r}=50 \mathrm{~Hz}$ and $m$ varies in the range of integers in $[0,599]$ and $n$ varies in the range of integers in $[1,40]$, the variation of harmonic amplitude as harmonic frequency $n w_{c} \pm m w_{r}$ changes is like Figure 4 . 


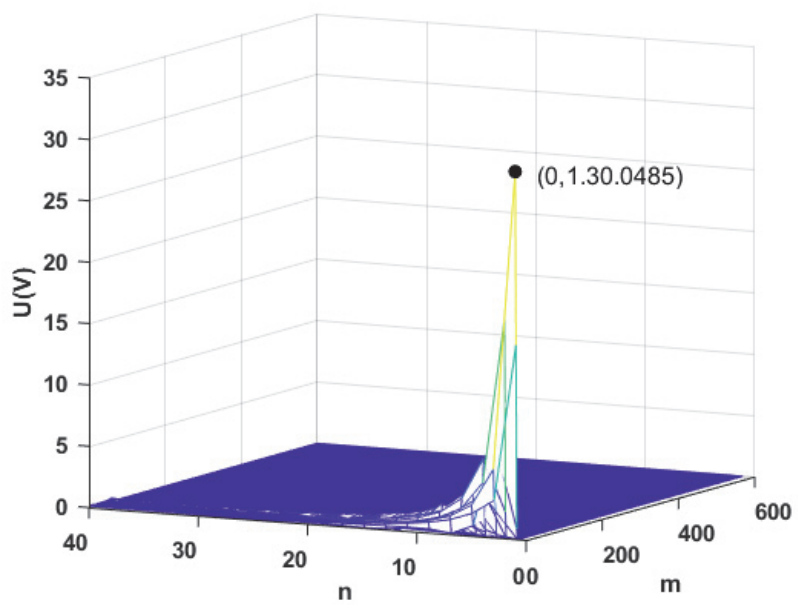

Figure 4. When $M=1$, the relationship between harmonic amplitude and harmonic frequency

From Figure 4, we see that harmonic component with the largest amplitude has an angular frequency of $w_{c}$. Harmonic components with amplitude greater than $5 \%$ of the fundamental amplitude $(2.5 \mathrm{~V})$ are listed in Table 1.

Table 1. Harmonic components with amplitude greater than $5 \%$ of the fundamental amplitude

\begin{tabular}{cccccc}
\hline Angular Frequency & $w_{c}$ & $2 w_{c} \pm w_{r}$ & $w_{c} \pm 2 w_{r}$ & $3 w_{c} \pm 4 w_{r}$ & $4 w_{c} \pm 3 w_{r}$ \\
\hline Amplitude (V) & 30.0485 & 18.0426 & 15.8965 & 7.8609 & 6.4594 \\
Angular Frequency & $3 w_{c}$ & $4 w_{c} \pm w_{r}$ & $5 w_{c} \pm 6 w_{r}$ & $4 w_{c} \pm 5 w_{r}$ & $6 w_{c} \pm 7 w_{r}$ \\
Amplitude (V) & 5.6417 & 4.4827 & 4.4046 & 3.5494 & 3.3042 \\
Angular Frequency & $3 w_{c} \pm 2 w_{r}$ & $6 w_{c} \pm 3 w_{r}$ & $5 w_{c} \pm 8 w_{r}$ & $5 w_{c}$ & $7 w_{c} \pm 10 w_{r}$ \\
Amplitude (V) & 3.1050 & 3.0547 & 2.6632 & 2.6008 & 2.5482 \\
\hline
\end{tabular}

Among the components in Table 1, the frequency of component with the largest amplitude is $w_{c}$. Because $w_{c}$ is much larger than $w_{r}$, the minimum frequency among these components is $w_{c}-2 w_{r}$. To effectively suppress harmonic components with amplitude greater than $5 \%$ of the fundamental amplitude, the low-pass filter is required to effectively eliminate components with frequency larger than or equal to $w_{c}-2 w_{r}$. So the cut-off frequency of the low-pass filter $w_{0}$ should be less than $w_{c}-2 w_{r}$ and also larger than fundamental frequency $w_{r}$, in order to prevent fundamental component from attenuation because of the filter. The range of $w_{0}$ is described in formula 9.

$$
w_{r}<w_{0}<w_{c}-2 w_{r}
$$

\subsubsection{Constraint of Filtering Effect of Low-pass Filter}

As for single-phase bipolar SPWM, given modulation depth and frequency modulation index adjusted, harmonic components of output voltage are still large relative to fundamental frequency, thus the quality of output voltage is low. Therefore, filtering high order harmonics with low-pass filter to improve the quality of output voltage is necessary. A common low-pass filter is LC low-pass filter whose circuit is showed in Figure 5. 


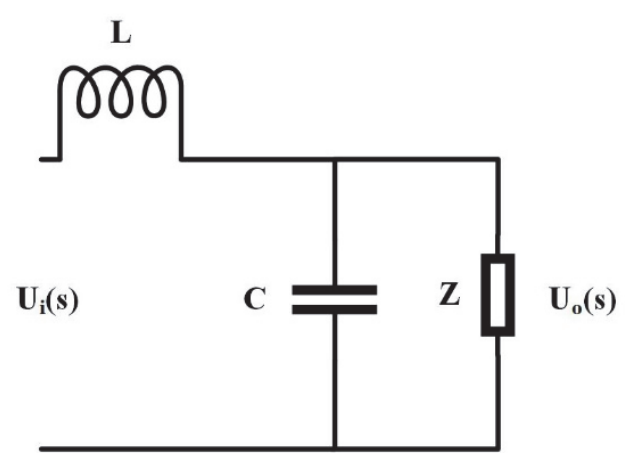

Figure 5. LC low pass filter circuit

In Figure 5, $Z$ refers to the load.

We research on resistive load and work out the transfer function in Formula 10.

$$
\frac{U_{o}(s)}{U_{i}(s)}=\frac{1}{L C S^{2}+\frac{1}{Z} \sqrt{\frac{L}{C}} \sqrt{L C} S+1}
$$

So, the cut-off frequency $w_{0}=1 / \sqrt{L C}$ (Song, Liu, Yan \& Chen, 2003).

In order to prevent fundamental component in input voltage from attenuation because of the filter, let $W(s)=20 \lg \left|U_{o}(s) / U_{i}(s)\right|$. Assuming that when $w=w_{r}$, the amplitude difference between output voltage $U_{o}$ and input voltage $U_{i}$ will not surpass $k_{1}$ times of input voltage amplitude $U_{i}$, we have

$$
\Delta W\left(j w_{r}\right)=|20 \lg | \frac{U_{o}\left(j w_{r}\right)}{U_{i}\left(j w_{r}\right)}|-20 \lg 1| \leq|20 \lg | 1 \pm k||
$$

Also, with low-pass filter, harmonics with frequency larger than $w_{c}-2 w_{r}$ are required to be fully suppressed. As a result, the amplitude of harmonics with frequency larger than $w_{c}-2 w_{r}$ is not larger than $k_{2}$ times of that before filtering.

$$
20 \lg \left|\frac{U_{o}\left[j\left(w_{c}-2 w_{r}\right)\right]}{U_{i}\left[j\left(w_{c}-2 w_{r}\right)\right]}\right| \leq 20 \lg k_{2}
$$

\subsubsection{Constraint of System Power Factor}

To assure a high system power factor after adding a filter in inverter circuit, we take system power factor as indicator and determine the parameters L, C in LC low-pass filter (Jiaju, Yiguo, Jilin \& Jianwei, 2003).

With a LC low-pass filter, system power factor $\cos \theta$ is as follow.

$$
\cos \theta=\frac{Z}{\sqrt{Z^{2}+\left[L w_{b}+L\left(Z C w_{b}\right)^{2} w_{b}-Z^{2} C w_{b}\right]^{2}}}
$$

And system power factor of the inverter should be not less than $k_{3}$.

$$
\cos \theta=\frac{Z}{\sqrt{Z^{2}+\left[L w_{b}+L\left(Z C w_{b}\right)^{2} w_{b}-Z^{2} C w_{b}\right]^{2}}} \geq k_{3}
$$

\subsubsection{Establishment of Mixed Integer Nonlinear Programming Model}

On condition that low-pass filter can effectively suppress harmonic components with frequency greater than $w_{c}-2 w_{r}$ and the attenuation of fundamental components is very small, that is, formula 11 and 12 are satisfied, and we find a cut-off frequency which is able to effectively suppress harmonics. However, the switching loss of IGBT is factor influencing harmonic suppression that cannot be ignored. IGBT's switching loss is direct 
proportional to IGBT's switching frequency so that IGBT's switching frequency should be controlled when suppressing harmonics in order to reduce loss. Therefore, we take frequency modulation index $P$ as objective function and formula $9,11,12,14$ as constraints to build a mixed integer nonlinear programming model as formula 15.

$$
\begin{aligned}
& \min P \\
& \text { s.t. }\left\{\begin{array}{l}
w_{r}<w_{0}<w_{c}-2 w_{r} \\
20 \lg \left|\frac{U_{o}\left(j w_{r}\right)}{U_{i}\left(j w_{r}\right)}\right|-20 \lg 1|\leq| 20 \lg \mid 1 \pm k \| \\
20 \lg \left|\frac{U_{o}\left[j\left(w_{c}-2 w_{r}\right)\right]}{U_{i}\left[j\left(w_{c}-2 w_{r}\right)\right]}\right| \leq 20 \lg k_{2} \\
\cos \theta=\frac{Z}{\sqrt{Z^{2}+\left[L w_{b}+L\left(Z C w_{b}\right)^{2} w_{b}-Z^{2} C w_{b}\right]^{2}}} \geq k_{3} \\
w_{0}=\frac{1}{\sqrt{L C}} \\
w_{c}=P w_{r} \\
0 \leq P \leq 600 \\
P \text { is a interger }
\end{array}\right.
\end{aligned}
$$

According to formula 15 , after we determine $k_{1}, k_{2}, k_{3}$ depending on the demand of output voltage, we can work out frequency modulation index $P$ and LC low-pass filter's parameters and suppress harmonics while controlling switching loss. Mixed integer nonlinear programming is a kind of NP-complete decision problem which takes a large amount of calculations to be solved. Many methods of solving mixed integer nonlinear programming problem find it hard to get the global optimal solution (Wu, Wang \& Chen, 2007). Scholars use various algorithms to solve this problem, such as Particle Swarm Optimization for Composite Particle (PSO-CP) (De, Kumar, Gunasekaran \& Tiwari, 2017) and mixed-integer hybrid differential evolution (MIHDE) (Lin, Hwang, Wang, 2004). In this article, we use the widely-used genetic algorithms which is easy to find the global optimal solution.

Based on the ideas of natural selection and genetics, genetic algorithms use genetic mutation, genetic recombination and other genetic mechanisms to search for optimal solution (Guo, Wang \& Han, 2010). The main steps of genetic algorithms are as follow.

Step 1: Determine initial parameter and initial group of population.

Step 2: Access the fitness of individuals in the group.

Step 3: Select two individuals as father and mother according to fitness.

Step 4: Crossover their homologous chromosomes with a certain probability and then generate offspring.

Step 5: Mutate offspring's homologous chromosomes with a certain probability.

Step 6: Repeat Step 2 to 4 until the optimal fitness has reached a plateau in successive iterations from parent generation to offspring generation.

With mixed integer nonlinear programming model established, we use genetic algorithm to first find the global optimal solution within real numbers and then work out the optimal integer solution near the optimal real number solution. In this way, we finally find the solution of mixed integer nonlinear programming.

\section{Simulation Verification and Discussion}

Using this harmonic suppression strategy, we simulate the single-phase bipolar SPWM inverter and compare the output voltage before suppression with that after to verify the correctness of this strategy.

\subsection{SPWM Simulation before Applying Harmonic Suppression Strategy}

When simulating single-phase bipolar SPWM with MATLAB/SIMULINK, the setting of values is that discrete sample time equals $10^{-7} \mathrm{~s}$, simulation time equals $0.24 \mathrm{~s}$, fundamental frequency equals $50 \mathrm{~Hz}$, amplitude modulation depth $M=0.6$, frequency modulation index $P=10$, load resistance $R=10 \Omega$, DC voltage $U_{d}=50 \mathrm{~V}$ and the largest switching frequency of IGBT is not larger than $30 \mathrm{kHz}$. 


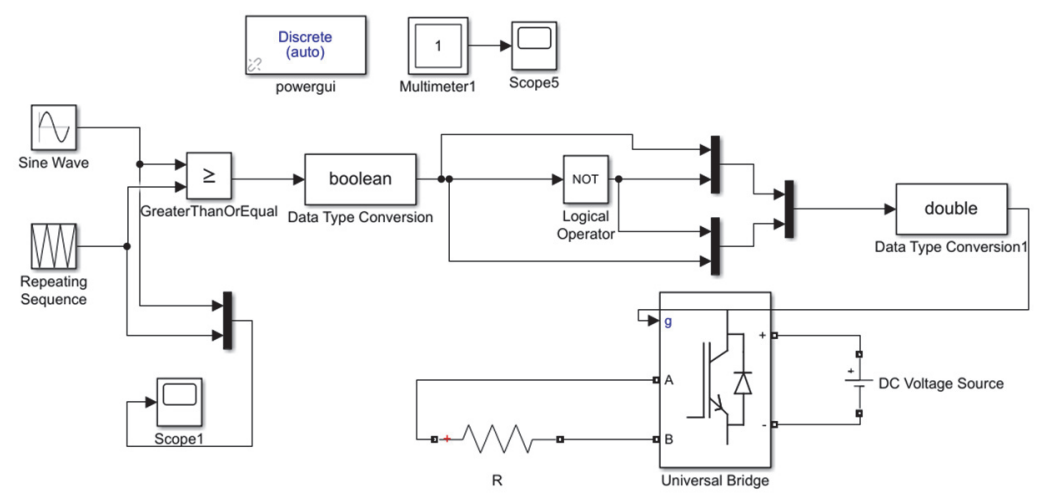

Figure 6. Single-phase bipolar SPWM original simulation circuit based on SIMULINK

The simulated wave of output voltage is showed in Figure 7.

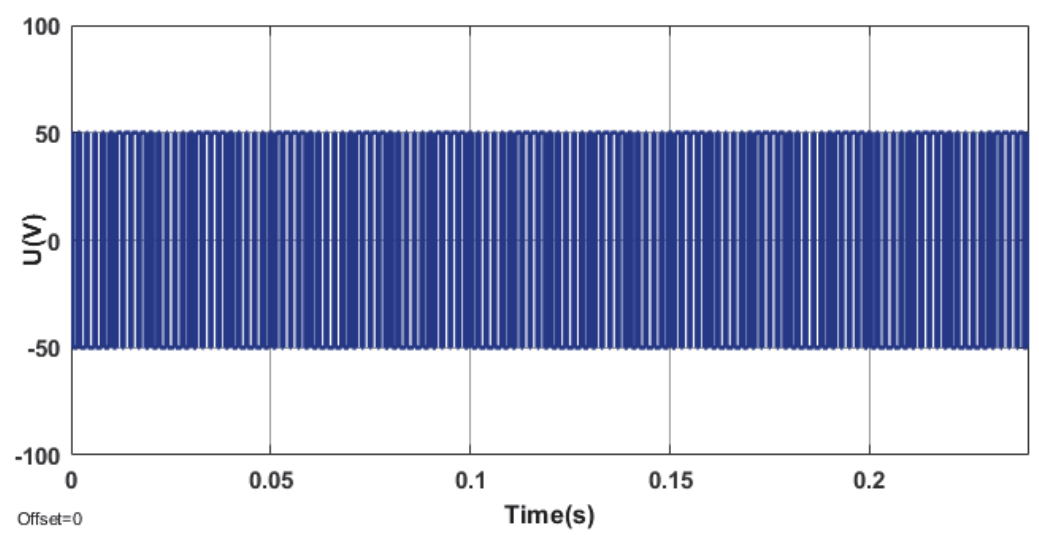

Figure 7. Output voltage waveform without using harmonic suppression strategy

Then we use Powergui to analyze output voltage wave with FFT Analysis and get the output voltage spectrum in Figure 8 . We infer from Figure 8 that the total harmonic distortion is $213.47 \%$ and fundamental amplitude is $29.99 \mathrm{~V}$. And harmonics with different amplitudes distribute dispersedly within different frequency bands. Among all harmonic components, component with a frequency of $500 \mathrm{~Hz}$ (angular frequency is $w_{c}$ ) is the largest harmonic component, whose amplitude reaches $167.66 \%$ of the fundamental amplitude.

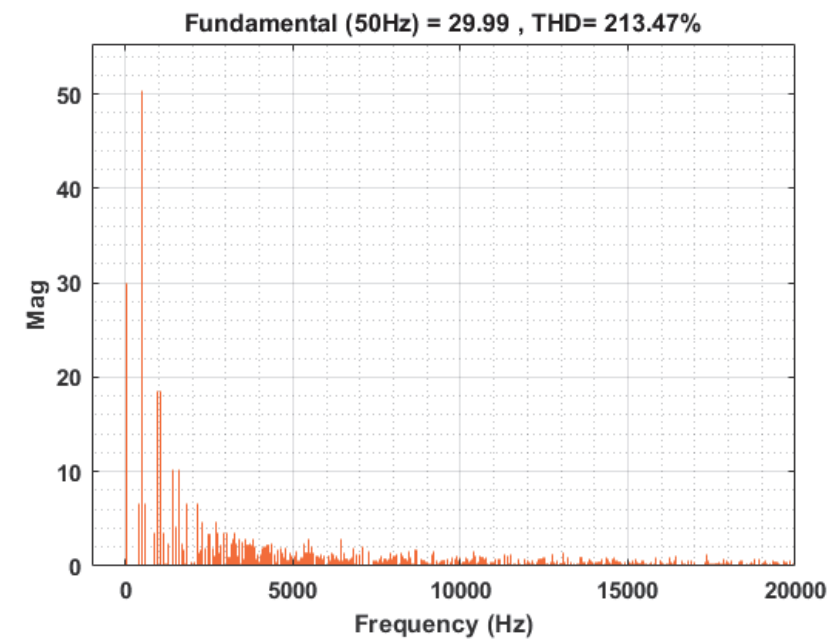

Figure 8. Spectrum analysis of output voltage without using harmonic suppression strategy 


\subsection{SPWM Simulation after Applying Harmonic Suppression Strategy}

According to our requirements, after harmonic suppression, the fundamental amplitude difference between output voltage $U_{o}$ and input voltage $U_{i}$ should not surpass $1 \%$ of the fundamental amplitude of input voltage $U_{i}$. And we require that the amplitude of each harmonic component with a frequency greater than or equal to $w_{c}-2 w_{r}$ to decline to no larger than $0.01 \%$ of the initial amplitude while system power factor is not less than 0.95 . So we can set $M=1, k_{1}=0.01, k_{2}=0.01 \%, k_{3}=0.95$. To use genetic algorithm, we set the population size as 500, mutation probability as 0.1 , crossover probability as 0.6 and the maximum number of generations as 200 , and then generate the optimal solution of $P$ in real number domain. The optimal solution is showed in formula 16 .

$$
\left\{\begin{array}{l}
w_{0}=669.4179 \mathrm{rad} / \mathrm{s} \\
P=215.0840 \\
L=19.4217 \mathrm{mH} \\
C=114.8995 \mathrm{uF}
\end{array}\right.
$$

In real number domain, 215.0840 is the optimal solution of $P$. So the frequency modulation index should be adjusted within the range $216 \leq P \leq 600$. Then take the last generation population in last process as the initial population to do genetic algorithm calculation one more time and get the results in formula 17.

$$
\left\{\begin{array}{l}
w_{0}=671.9660 \mathrm{rad} / \mathrm{s} \\
P=216 \\
L=19.3641 \mathrm{mH} \\
C=114.3691 \mathrm{uF}
\end{array}\right.
$$

Here $P=216$ is the optimal solution. Substituting the results in formula 17 into formula 13, we work out that power factor $\cos \theta=0.9503$, which satisfy the requirement that inverter system power factor should not less than 0.95 . Modify SIMULINK-based simulation circuit according to parameter(17) after solution and get circuit in Figure 9.

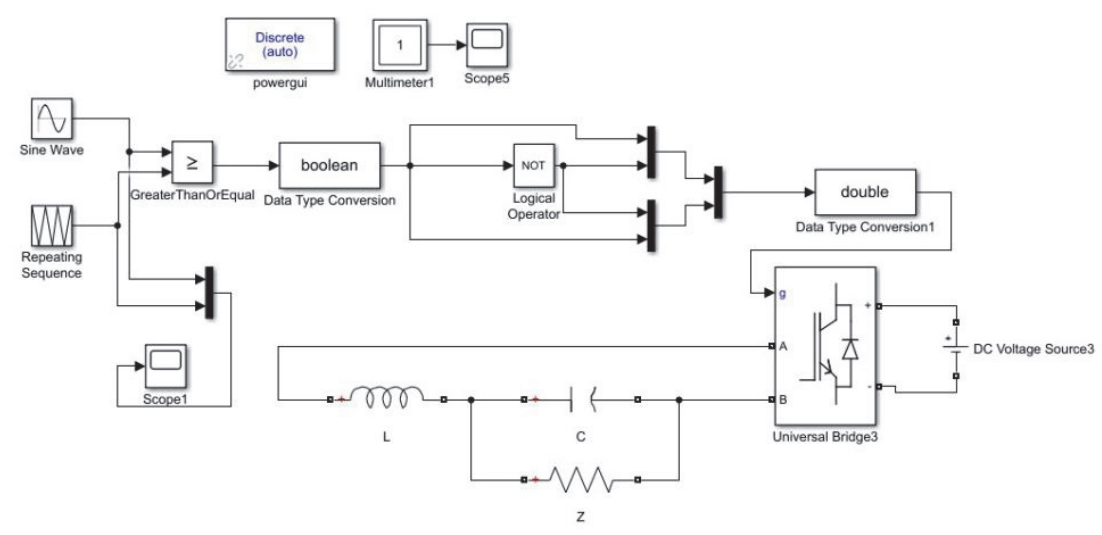

Figure 9. Modified SIMULINK-based simulation circuit

Set simulation time as $0.24 \mathrm{~s}$ and get the output voltage wave in Figure 10. 


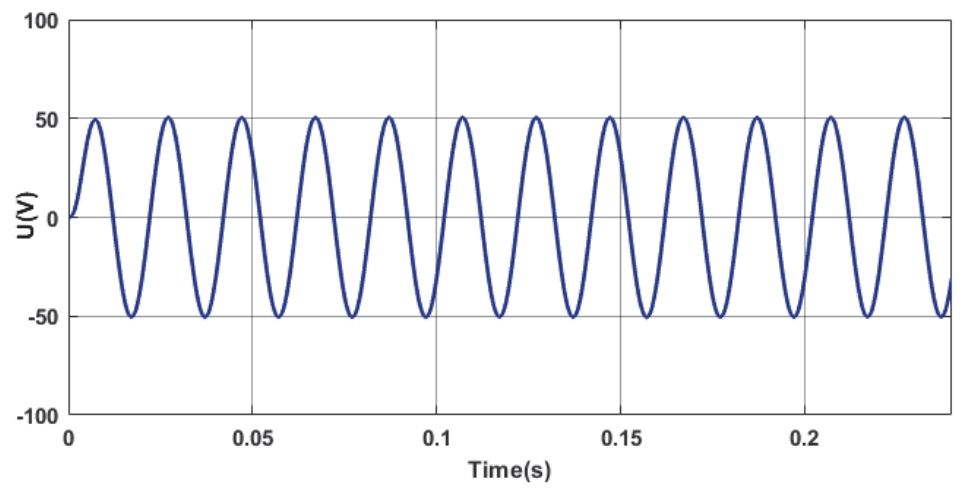

Figure 10. Output voltage waveform using harmonic suppression strategy

We see that after filtering, the output voltage waveform shows the shape of a sine wave whose contour is clear and curve is smooth, and the output voltage quality is greatly improved compared to the case where harmonic suppression is not performed. Then we use Powergui to perform FFT Analysis on the stable waveform of the output voltage and get the spectrum analysis in Figure 11.

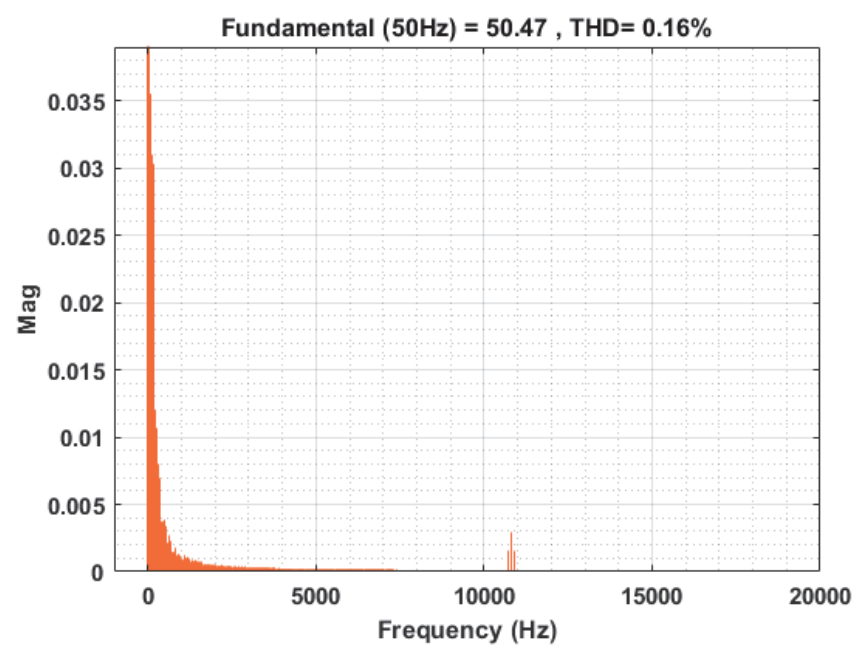

Figure 11. Spectrum analysis of output voltage using harmonic suppression strategy

Figure 11 shows that the total harmonic distortion of the output voltage waveform is equal to $0.16 \%$, which is 99.93\% less than the total harmonic distortion when harmonic suppression is not performed. And we see that harmonic components mainly distribute in low frequency band because nearly all components with high frequency are effectively eliminated after applying this suppression strategy and quality of output voltage is improved. And the fundamental amplitude of output voltage $U_{b}$ is equal to $50.47 \mathrm{~V}$, which change at a rate of $0.94 \%$ that is less than required $k_{1}=1 \%$, compared to the fundamental amplitude of input voltage. Harmonic components with an initial amplitude larger than $5 \%$ of the fundamental amplitude $(2.5 \mathrm{~V})$ and their amplitudes after harmonic suppression are listed in Table 2.

Table 2. Harmonic components with an initial amplitude greater than $5 \%$ of the fundamental amplitude after harmonic suppression

\begin{tabular}{cccccccc}
\hline Angular Frequency & $w_{c}$ & $2 w_{c}-w_{r}$ & $2 w_{c}+w_{r}$ & $w_{c}-2 w_{r}$ & $w_{c}+2 w_{r}$ & $3 w_{c}-4 w_{r}$ & $3 w_{c}+4 w_{r}$ \\
\hline Amplitude (mV) & 2.9340 & 0.2592 & 0.1834 & 1.5760 & 1.5210 & 0.0996 & 0.0976 \\
Angular Frequency & $4 w_{c}-3 w_{r}$ & $4 w_{c}+3 w_{r}$ & $3 w_{c}$ & $4 w_{c}-w_{r}$ & $4 w_{c}+w_{r}$ & $5 w_{c}-6 w_{r}$ & $5 w_{c}+6 w_{r}$ \\
Amplitude $(\mathbf{m V})$ & 0.0167 & 0.0214 & 0.0544 & 0.0376 & 0.0117 & 0.0131 & 0.0124 \\
\hline
\end{tabular}




\begin{tabular}{cccccccc}
\hline Angular Frequency & $4 w_{c}-5 w_{r}$ & $4 w_{c}+5 w_{r}$ & $6 w_{c}-7 w_{r}$ & $6 w_{c}+7 w_{r}$ & $3 w_{c}-2 w_{r}$ & $3 w_{c}+2 w_{r}$ & $6 w_{c}-3 w_{r}$ \\
Amplitude (mV) & 0.0234 & 0.0518 & 0.0191 & 0.0102 & 0.0507 & 0.0498 & 0.0114 \\
Angular Frequency & $6 w_{c}+3 w_{r}$ & $5 w_{c}-8 w_{r}$ & $5 w_{c}+8 w_{r}$ & $5 w_{c}$ & $7 w_{c}-10 w_{r}$ & $7 w_{c}+10 w_{r}$ & - \\
Amplitude (mV) & 0.0143 & 0.0112 & 0.0107 & 0.0111 & 0.0155 & 0.0153 & - \\
\hline
\end{tabular}

Table 2 shows that harmonics with amplitude larger than $5 \%$ of the fundamental amplitude $(2.5 \mathrm{~V})$ before harmonic suppression, have decayed to less than $0.1 \%$ of the original which meets the demand of harmonic suppression of output voltage.

Therefore, the simulation results show that after using the harmonic suppression strategy, the output voltage quality is greatly improved and the harmonic components are effectively reduced while a high power factor of the system is obtained. With the simulation experiment, the correctness of this harmonic suppression strategy is verified.

\section{Conclusions}

This article researches on single-phase SPWM bipolar inverter output voltage, establishes a mathematical model of the output voltage to examine the influence of amplitude modulation depth, frequency modulation index and LC low-pass filter's parameter on output voltage's harmonics and finally formulates harmonic suppression strategy in single-phase SPWM bipolar inverter's output voltage. At first, we work out the mathematical form of singlephase bipolar SPWM inverter output voltage based on SPWM theory and Fourier series theory. Through calculation and graphical analysis, we identify that harmonic components with frequency equal to carrier frequency accounts for the largest and has the greatest impact on the quality of output voltage. Then we research on the relationship between amplitude modulation depth and output voltage's fundamental amplitude and also the relationship between amplitude modulation depth and output voltage's harmonic amplitude by adjusting amplitude modulation depth. The conclusion is that increasing amplitude modulation depth helps increasing fundamental wave while reducing harmonics. And when amplitude modulation depth is set as 1 , the effect of harmonic suppression comes out the best. So we fix amplitude modulation depth as 1 and analyze harmonics with amplitude greater than $5 \%$ of the fundamental amplitude. To fully suppress these harmonics which significantly affect the quality of output voltage, we use LC low-pass filter and also reduce switching loss by controlling frequency modulation index while keeping a modest fundamental loss and a high system power factor. To figure out the global optimal solution of frequency modulation index and inductance and capacitance of the filter, we establish a mixed integer nonlinear model and use genetic algorithm to search for two times. Finally, after simulation verification in SIMULINK, we find that the total harmonic distortion in single-phase SPWM inverter output voltage was reduced from $213.47 \%$ to $0.16 \%$, thus the quality of output voltage is improved greatly. The simulation proves the effectiveness of our harmonic suppression strategy, which offers ideas for harmonic elimination in single-phase SPWM inverter output voltage in reality.

Due to space limitations, this research still has defects in perspective and method of research when researching on single-phase SPWM harmonic suppression. Firstly, to restrict the switching loss of IGBT, we discuss the influence of switching frequency but ignore the switching voltage and current. Secondly, we verify the results by simulating with SIMULINK instead of by hardware designing. So, the results of simulation may have some errors.

However, harmonic suppression strategy put forward in this article has distinct advantages. We first analyze the distribution of harmonics to find out the most influential harmonic components so that a harmonic suppression strategy based on this analysis can be more effective. What's more, we establish a mixed integer nonlinear model to adjust the strategy so that switching loss can be reduced at the same time of ensuring harmonic suppression and improving system power factor. Therefore, the harmonic suppression strategy in this article has a practical significance in applications and serves as a reference for single-phase SPWM inverter harmonic suppression.

\section{References}

Blanco, E. L. (2017, September). Design and implementation of an inverter with SPWM modulation. In 2017 IEEE Central America and Panama Student Conference (CONESCAPAN)(pp. 1-6). IEEE. https://doi.org/10.1109/CONESCAPAN.2017.8277608

De, A., Kumar, S. K., Gunasekaran, A., \& Tiwari, M. K. (2017). Sustainable maritime inventory routing problem with time window constraints. Engineering Applications of Artificial Intelligence, 61, 77-95. https://doi.org/10.1016/j.engappai.2017.02.012

Fengjun, L. (2002). Sinusoidal Inverter. Beijing: Science Press. (In Chinese) 
Guo, P., Wang, X., \& Han, Y. (2010, October). The enhanced genetic algorithms for the optimization design. In 2010 3rd International Conference on Biomedical Engineering and Informatics (Vol. 7, pp. 2990-2994). IEEE.https://doi.org/10.1109/BMEI.2010.5639829

Hanchao, Z., \& Daolian, C. (2017, October). A single-stage isolated charging/discharging DC-AC converter with second harmonic current suppression in distributed generation systems. In IECON 2017-43rd Annual Conference of the IEEE Industrial Electronics Society (pp. 4427-4432). IEEE. https://doi.org/10.1109/IECON.2017.8216762

Hannan, S., Aslam, S., \& Ghayur, M. (2018, February). Design and real-time implementation of SPWM based inverter. In 2018 International Conference on Engineering and Emerging Technologies (ICEET) (pp. 1-6). IEEE. https://doi.org/10.1109/ICEET1.2018.8338637

Jilin, W. J. Z. Y. R., \& Jianwei, A. (2003). A Method to Design of Filter for Single-phase PWM Inverter. Electric Drive, (3), 2. https://doi.org/10.19457/j.1001-2095.2003.03.003

Lin, W. (2006, June). A new approach to the harmonic analysis of SPWM waves. In 2006 International Conference on Mechatronics and Automation (pp. 390-394). IEEE. https://doi.org/10.1109/ICMA.2006.257565

Lin, Y. C., Hwang, K. S., \& Wang, F. S. (2004). A mixed-coding scheme of evolutionary algorithms to solve mixedinteger nonlinear programming problems. Computers \& Mathematics with Applications, 47(8-9), 1295-1307. https://doi.org/10.1016/S0898-1221(04)90123-X

Rahman, H., Javed, M. Y., Saleem, Y., Ling, Q., \& Gulzar, M. M. (2018). Cascaded Hybrid Multi-level Inverter for Selective Harmonics Elimination. Iranian Journal of Science and Technology, Transactions of Electrical Engineering, 42(2), 135-148. https://doi.org/10.1007/s40998-018-0054-8

Song, Q., Liu, W., Yan, G., \& Chen, Y. (2003). LC filter design for high-power PWM voltage source inverter. Qinghua Daxue Xuebao/Journal of Tsinghua University (China), 43(3), 345-348. https://doi.org/10.16511/j.cnki.qhdxxb.2003.03.016

Wu, L. H., Wang, Y. N., \& Chen, Z. L. (2007). Modified Differential Evolution Algorithm for Mixed-integer Nonlinear Programming Problems. MINIMICRO SYSTEMS-SHENYANG-, $28(4), 666$. https://doi.org/10.3969/j.issn.1000-1220.2007.04.019

Wu, Y., Song, X., Li, H., \& Chen, B. (2018). Suppression of harmonic current in permanent magnet synchronous motors using improved repetitive controller. Electronics Letters, 55(1), 47-49. https://doi.org/10.1049/el.2018.7035

Xu, S. G., Xu, J., \& Cao, T. (2010). Analysis and research of SPWM inverter harmonic suppression [J]. Journal of University of Electronic Science and Technology of China, 39(5), 701-705. http://doi.org/10.3969/j.issn.1001-0548.2010.05.012

Zhaoan, W., \& Jun, H. (2000). Power Electronics Technology. Beijing: China Machine Press. (In Chinese)

Zhou, K. L., Lin, Z. H., \& Han, Z. G. (2003). Realization of Sinusoidal Pulse-Width-Modulation Signal Based on Harmonic-Suppression [J]. Journal of Shanghai Jiaotong University, 9. http://doi.org/10.16183/j.cnki.jsjtu.2003.09.015

\section{Copyrights}

Copyright for this article is retained by the author(s), with first publication rights granted to the journal.

This is an open-access article distributed under the terms and conditions of the Creative Commons Attribution license (http://creativecommons.org/licenses/by/4.0/). 